\title{
Decision-Making BIM Platform for Chemical Building Products
}

\author{
Gabriele Gazzaniga, Luigi Coppola, Bruno Daniotti, Claudio Mirachi, \\ Alberto Pavan and Valeria Savoia
}

\begin{abstract}
This paper presents the innovative solution based on BIM technology proposed to BASF Construction Chemicals Italia for the digitization of chemical products for buildings. It deals with the implementation of a system able to support stakeholders during the selection phase and with the digitization of product information without geometrical features such as concrete admixtures or repair products. The most significant problems linked to the construction sector are deep fragmentation and the characterizing inefficiency of the building process; these phenomena are often due to uniqueness, unevenness, and inconsistency and they result in quality decay, lengthening of working time and claims. The construction sector needs properties like dynamicity and a structured and easily consultable database in order to reorganize information. BIM could represent the solution to these requirements since it is able to manage knowledge and information among the involved stakeholders and manufacturers during the building process and the useful life of a building itself. Thanks to the digitization of information made possible by the BIM process, it is possible to define the products' attributes that are key to informing the relevant stakeholders and to import them into a digital model in order to reduce inaccuracies during the constructive phases and to draw a guideline for a potential restoration.
\end{abstract}

Keywords Building information modeling - Chemical products for construction • Products without geometrical features

\footnotetext{
G. Gazzaniga $(\bowtie) \cdot$ L. Coppola

Department of Engineering and Applied Sciences, University of Bergamo, Bergamo, Italy e-mail: gabriele.gazzaniga@unibg.it
}

B. Daniotti · C. Mirachi · A. Pavan

Architecture, Built Environment and Construction Engineering_ABC Department, Politecnico di Milano, Milan, Italy

V. Savoia

BASF Construction Chemicals Italia Spa, Treviso, Italy 


\section{Introduction}

Despite the fact that during the last decade a lot of innovative proposals concerning constructive techniques, materials and project management have been presented, the construction sector has not always been able to incorporate these innovations (Taxén and Lilliesköld 2008). Differently to other industrial processes, the building process is strongly characterized by a historical component made of old traditions and techniques that inexorably conflict with the available innovations that have been usually seen as negative only because they are not aligned with those always used. Another problem related to the construction sector is project management (Clough et al. 2008): many different figures have to cooperate; this lack is getting bigger due to the uniqueness and the unevenness that are typical of the building process (Kifokeri and Xenidis 2017).

A new instrument capable of managing all the different information and of establishing a positive communication system between the different stakeholders is just what the construction sector needs. Property of dynamism and immediacy are typical of Building Information Modeling. Initially BIM has been seen as a new ComputerAided Design (CAD) able to represent in a better way the different project in terms of 3D (Succar 2009); nowadays, BIM is understood as an overarching variety of activities in an object-oriented dimension which supports the representation of building elements in terms of their 3D geometric and non-geometric attributes and relationship (Tookey 2017). BIM embodies a series of new technologies and solutions with the aim of an interorganizational collaboration among the different stakeholders to improve design, construction, and maintenance (Miettinen and Paavola 2014). According to the National Institute of Building Science, BIM is defined as "a digital representation of physical and functional characteristics of a facility and a shared knowledge resource for information about a facility forming a reliable basis for a decision during the project's life-cycle" (NIBS 2018). The main functionality of BIM is to create a database of information using all the inserted data which, concurrently with its $3 \mathrm{D}$ representation, could be able to improve design construction, prefabrication and the operations of a facility (Charehzehi et al. 2017). The innovative potential of BIM allows stakeholders to exploit different project levels (UNI 11337 2018): by connecting 3D geometric models to the schedule data it is possible to obtain a 4D aspect that is able to provide several efficient advantages in order to facilitate site planning and management. Another level of BIM technology is 5D which allows for the monitoring of costs during the constructive process; the 6D aspect describes the building in relation to the management of the building itself in terms of usage, administration, maintenance, and future disposal. Finally, the interest in sustainability and environmental impact is contained in the 7D aspect.

Although BIM has yet to be fully adopted for the reason mentioned above, a lot of studies have described the potential of this instrument (Tookey et al. 2018; Miettinen and Paavola 2014; NIBS 2018; Bryde et al. 2013). Following real examples of constructions manufactured with BIM technology, have analyzed the benefits of using BIM in relation to some important criteria such as cost, time, communication, 
coordination, quality, risk mitigation, organization, and relation to software and found out that all of these criteria have been positively mentioned especially in terms of cost reduction and time-saving.

With the introduction of BIM as a new powerful instrument for integrated design, a lot of different companies engaged within the construction sector have tried to introduce BIM applications in their business in order to allow designers and builders to operate with a BIM approach. Although this effort attempts to move in an innovative direction, a lot of inaccuracies and mistakes regarding data management are noticeable (Fu and Zhang 2014); the main deficiencies concern the possibility to modify or update certain data actively among the different stakeholders, the incorrect allocation of data about materials' composition which usually causes an unclear generalization and discrepancy between the data collected in the technical datasheets and the ones stored in BIM objects. These inaccuracies lead to confusing BIM libraries made up of BIM object with lacking information, moreover, if data is inserted using BIM authoring software without a logical system of organization, BIM objects cannot be used efficiently. BIM could become more effective only if the BIM object is connected with an external structured database to reduce the inaccuracies about information exchange, to integrate the BIM approach with the company's actual information management system and make it easier to upgrade the data.

\section{Current Italian Situation Regarding BIM Utilization}

The evolution over the past 3 years of the number of procedures that prevent the use of BIM is growing rapidly (OICE 2018): in 2015 the BIM calls were only 4, in 2016 they rose to 26 , in 2017 their number increased to 83 with an increase of $70 \%$. In 2018, the calls for applications for the BIM methodology were a staggering 268 with a leap of $229 \%$. The particularly positive trend recorded finds its reasons both in a weak economic recovery but above all in the changes contained in the code of public contracts (D. LGS 50/2016) which came into force in April 2016 and provided for the obligation to entrust the work on the basis of the executive project, bringing to the market shares of services that were included in integrated procurement. The growing trend in the calls for applications for the BIM methodology should be reinforced in the immediate future with the entry into force of the Ministry of Infrastructure and Transport decree governing the obligation to use BIM in the design of public works.

Another important parameter for a better understanding of the BIM dissemination during 2018 is the analysis of the distribution of calls in which the BIM methodology is required for the number of activities entrusted. The "design activity" item alone represents about half of the total types of assignments, followed by the object's "seismic and structural safety assessment". A peculiarity that emerges from these analyses and that is linked to the object's "construction supervision", is, in fact, that in terms of numbers it covers a percentage equal to $1.5 \%$ while in terms of the economic value it reaches up to $15 \%$. This discrepancy between the two parameters can be interpreted as an effort by the client to entrust the BIM methodology with 
great importance in terms of operations management, in order to underline the great potential of this instrument.

The last interesting aspect concerns the destination of the type of contracting authority; in light of what has been previously stated, it is easy to expect that most of the contracts to be carried out using the BIM methodology are under the administration of the state which alone covers more than $50 \%$ of the allocations. Other institutional forms, such as municipalities and provinces, give a fair relevance to the methodology while for private tenders the figure settles around a value equal to $3.7 \%$.

\section{The Information Management Process for Chemical Products in a BIM-Based Approach}

The powerful aspect of projects implemented by BIM technology lies in an accurate representation of different construction elements and in a rich database accompanying the element itself; BASF Construction Chemicals Italia, international leader in the production of different types of chemical products, wants to implement a new process able to digitize all the information linked to construction materials without precise geometrical features for which until today it has been difficult to find a precise allocation within the BIM panorama for difficulties relating to an effective representation (Fig. 1).

The first step of this study concerns the choice of the most appropriate product and its attributes to digitize; starting from an accurate analysis of the company's entire portfolio, it was necessary to expand on two consequential points. First, tree diagrams have been defined to follow a Fault Tree Analysis in order to reproduce the logical process that leads to the manifestation of a need for the choice of product. Fault Tree

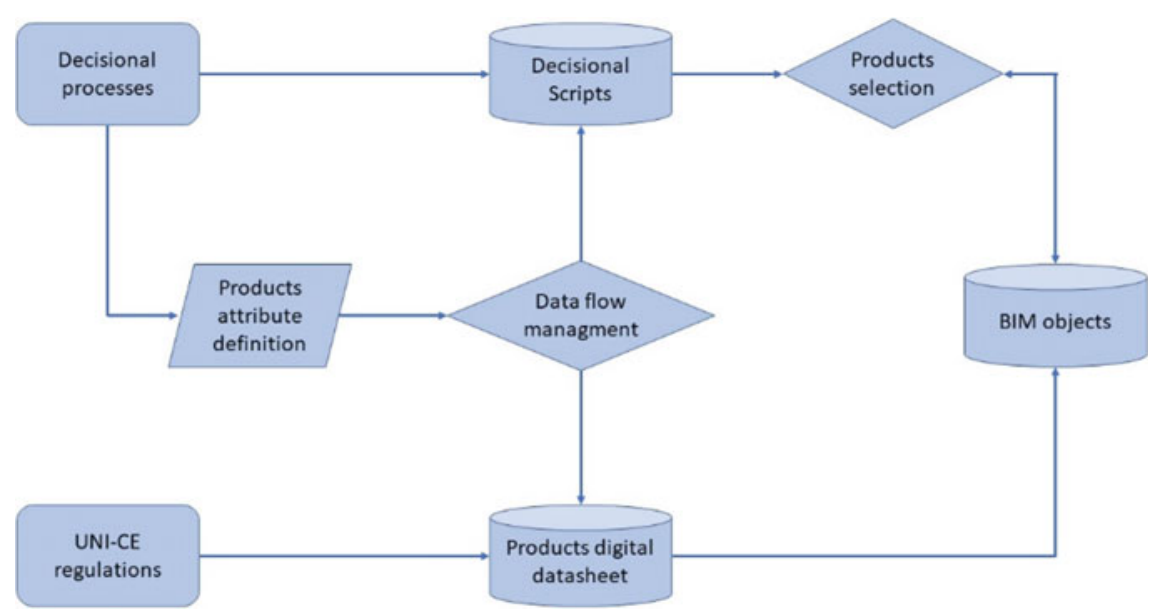

Fig. 1 Research method 
Analysis is a simple widespread model in the field of reliability and it can be analyzed by implementing the Binary Decision Diagram; BDD converts the fault tree model into a binary decision diagram which encodes an if-then-else structure that could be referred to a Boolean equation (Sinnamon and Andrews 1997). Second, going through the structured logical process and tracing back through the tree diagram, it is now possible to define unanimously the specific product or the particular construction technique thanks to the use of iterative decisional scripts; these are nothing more than small applications that allow the user, by answering simple questions, to retrace their way through the Fault Tree diagram instantaneously up to the choice of the final product in order to digitize the information via a BIM-based approach (Fig. 2).

The first group of construction materials addressed is the concrete admixtures; these chemical products are one of the components of concrete mix design with cement, aggregates, water, and possible additions. UNI EN 934-2 (UNI EN 9342 2012) brings together every type of admixture according to their main function. Nowadays, there are a lot of available concrete admixtures capable of ensuring the user with the expected performance related to mix design optimization in order to reduce water demand and consequently increase compressive strength or workability over time, or capable of managing particular operating needs since it could be necessary to intervene on the rheology side of particular cases and climatic and environmental conditions by giving concrete specific properties to prevent malicious events linked to chemically aggressive substances or external temperature. Once all the relationships have been established, a decisional datasheet based on a multi-criteria analysis is implemented in order to produce the definition of technical specifications automatically for a new mix design starting from the input provided by the users (Fig. 3).
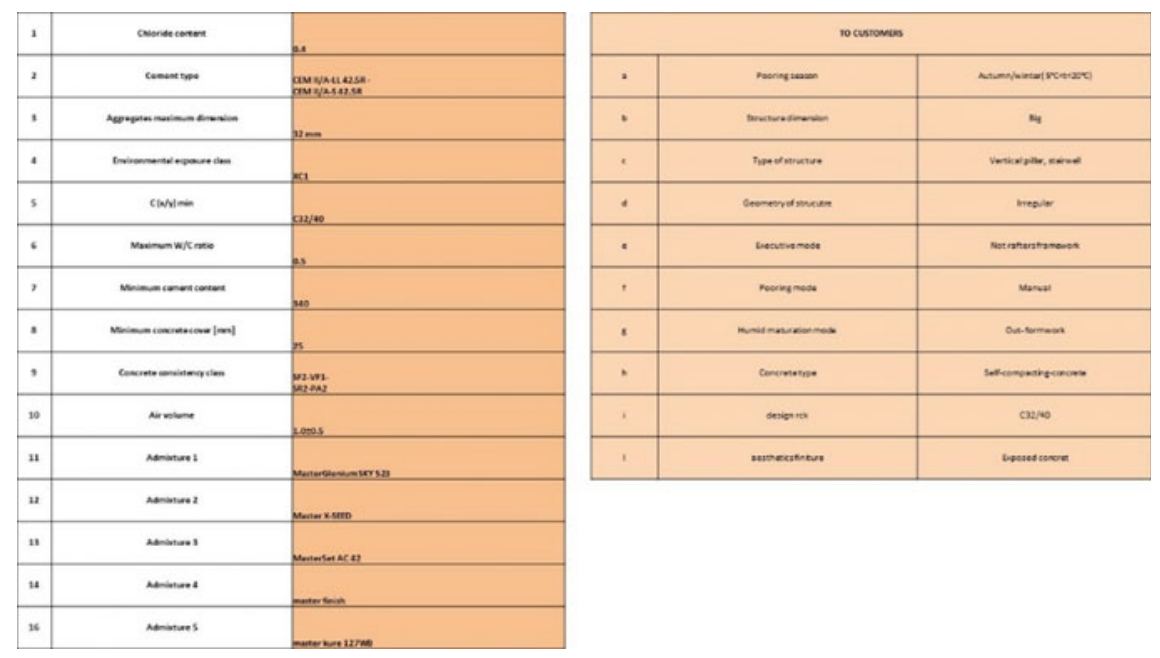

Fig. 2 Example of the decisional script 


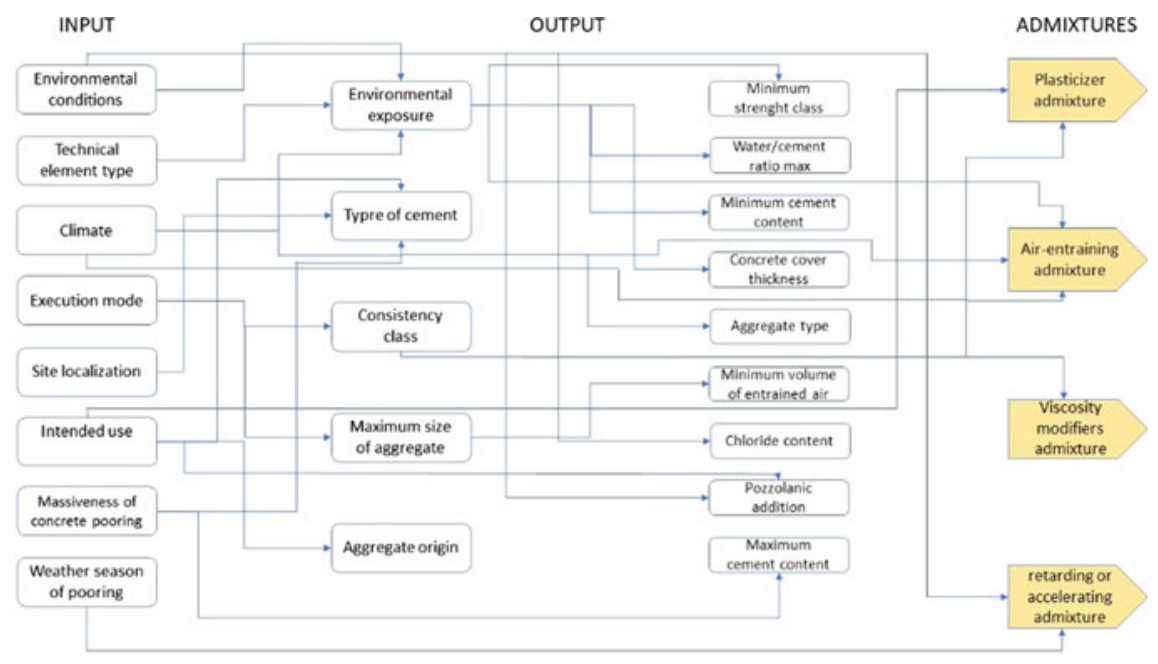

Fig. 3 Relationship requirements/boundary specifications for concrete attributes selecting

The second class of analyzed materials concerns restoration, maintenance, and reinforcement products (UNI EN 1504 2006). Differently from concrete admixtures, in addition to environmental and climatic condition, restoration and maintenance interventions depend on the failure mode and on its deterioration triggers. The decisional path to lead customers to a specific product and its correct placement starts from the potential causes and it is definitively set by combing fault tree knowledge results with other considerations about operating aspects and particular constructive techniques. Subsequently, decisional applications based on the decisional diagrams are implemented, filtering solutions on the basis of customer-compiled parameters and producing the most suitable product and even the tender specification (Fig. 4).

Proceeding with the analysis of construction materials the attributes of which have to be digitized, products for the protection and sealing of concrete surfaces have been investigated. The goal of these products is to create a superficial film that is able to avoid access into the cement matrix of substances that can promote degradation. Parameters which guide the choice of a product belonging to this category depend on the type of structure and on its function. A first distinction concerns the environment in which the object is placed; in fact, depending on the climatic situation, exposure to rain or other elements, different types of protection and sealing could be chosen; the second distinction concerns the type of structure and the function envisaged for the product; finally, operational needs must also be taken into consideration, such as the time of covering a product or putting it back into service. These systems are often composed of more than one product type, each of them with specific functions: first of all the primer guarantees a better adhesion to the substrate, it can vary according to the type of substrate and the conditions are the same; then there is the sealing membrane, which can be applied by spray or brush in a number of variable hands 


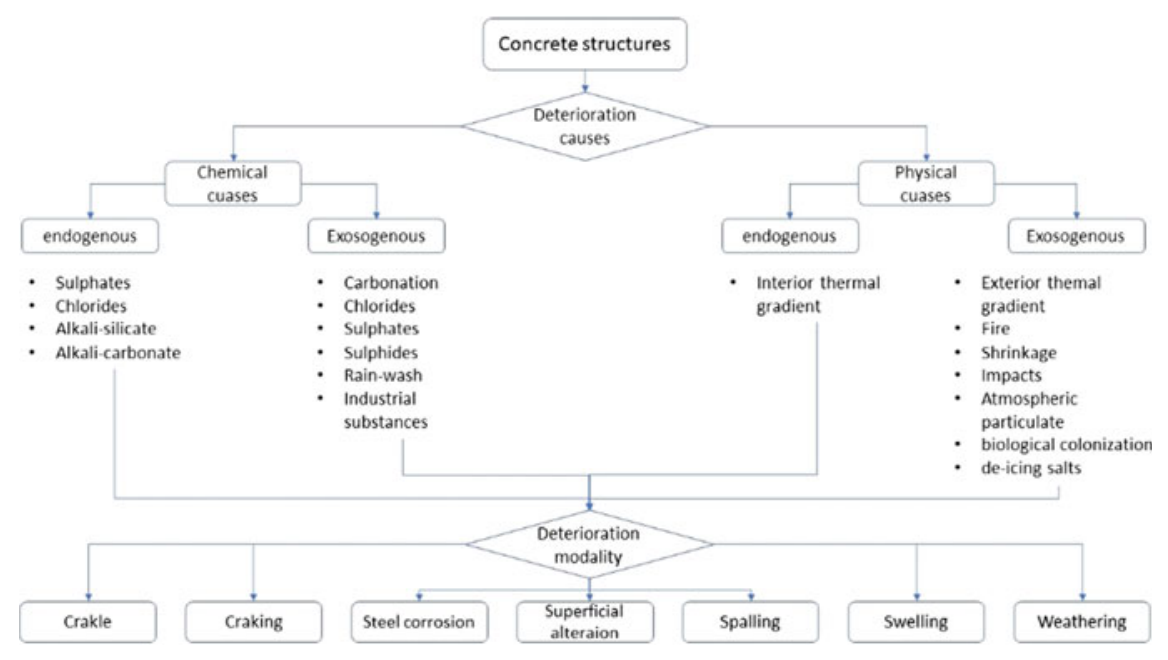

Fig. 4 Causes and types of failures in reinforced concrete structures

according to the aforementioned exposures, and finally the finish ensures a pleasant color rendering and an additional protection against UV rays.

The last category of analyzed products is the flooring finishes; environmental conditions, the position in the building, loads to be carried but also other specifications such as hygiene, health, and safety requirements determine the selection of the proper flooring solution. Precisely because of the vastness of the range of products available, it has been essential in the decision-making process to create a close relationship between the technical performance of the product, the customer's requests and the conditions of use; multicriteria analyses, that allow the stakeholders to choose the proper product for each case, start from the definition of importance values for each performance based on a relationship with decisional parameters, go through a normalization of measurement to obtain the ideal values and end with the calculation of the sequence to select the final product with a combination of importance values and normalized indicators (Cavalcante 2014). Similarly to what has been previously described for the protective and sealing products, the floor finishes also require the creation of a system composed of different elements: the primer creates a monolithic bond with the support, a coating made of reactive resin determines flooring mechanical properties, the filler spread on the fresh coating helps to improve the coating power on specific floor and finishing layers create a scratch-resistant surface and provide an aesthetic value.

Once all the decisional processes have been defined and digitized, the customer now has at their disposal a precise mix design or a series of products with the specific technical specifications according to their needs. At this point, all the information relating to the products that emerged from the previous step has to be translated into a BIM approach: to be able to do this, templates were created for each type of product. Templates are the BIM models to be used to start the design process and, by 
containing the data fields provided for the different product categories, can, therefore, receive BIM objects, virtualizations of geometric and non-geometric attributes of finite entities, physical or spatial, referred to a project, or to a complex of projects and to their processes. The digitization of the data of each product resulting from the decisional script is performed by a plug-in which takes care of the transfer of the above data to the related BIM objects present in the project creating a correspondence with an external database and the same BIM objects through a codification system (Fig. 5).

This new process for the digitization of objects without geometrical features, as well as allowing a punctual transfer of all information related to the product, provides the user with other potential uses in order to optimize both the design and executive phase. The first advantage that can be favored is the possibility of managing the project according to the different levels of development of the project (LOD); each type of profile will have particular information needs according to the functions carried out within the process: the designer will want to know the technical performance characteristics of the products, the company will also need information regarding the execution methods and the preconditions for installation, the manager will need instructions for maintenance. However, the building process, despite the multiplicity of actors and specializations involved, must lead to the creation of a unique product, and the uniqueness and the consistency of the choices during the various phases is an essential prerogative for quality in the final result. It was, therefore, decided to structure the information in such a way that the most frequently consulted data is directly allocated to the BIM model and, on the basis of this, the LODs have been determined in order to define, for each product category, the representative configurations of the BIM objects and the informative contents made immediately accessible on the model.

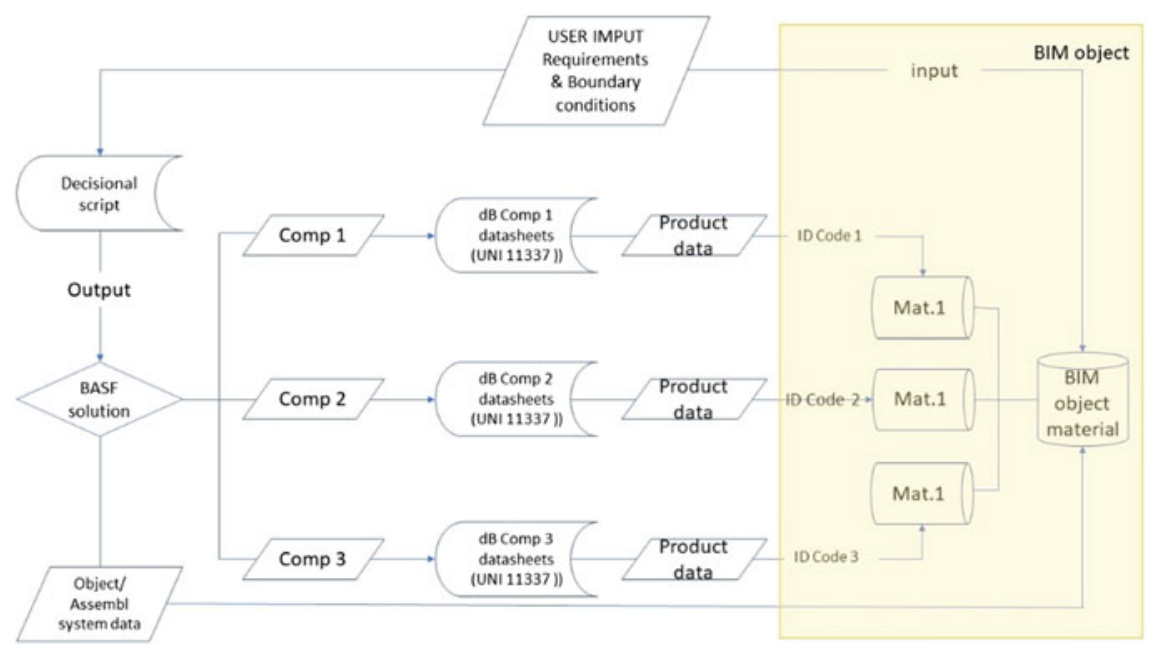

Fig. 5 General information flow for data digitization 
The second advantage is still linked to the optimization of the production process in relation to the different figures involved and this time concerns the prefabrication of the concrete. The correct realization of a mix design goes through a careful design process, a careful choice of admixture, a correct mixing, and finally an implementation carried out in a workmanlike manner; designer has to establish the class of exposure and the performance characteristics of the concrete mixture, the concrete producer has to choose the precise definition of the components making up the mixture, the dosages and the granulometric curve, the manufacturer of additives has to propose the right admixtures for the required performances and the executor is responsible for implementing the final product. This BIM approach allows users to put into communication the different figures involved in the prefabrication thanks to the drafting of a specific board, where there are specific items for each figure and for each step of the concrete packaging; at each step it is possible to make changes to this sheet and consequently update the database linked to the concrete in production and obtain from time to time the BIM objects that re congruent to the changes made.

The last advantage provided by this new digitization process is connected to ordinary and extraordinary maintenance works. Indeed, by offering the chance to create a complete model in which the replay operations made with regards to layers without any geometric properties can also be digitized, it is now possible to leave an unequivocal trace of every action performed on the structure; this new possibility granted by the digitization of these elements allows an in-depth knowledge of the product in the design phase which translates into a careful design that takes into account any previous interventions and that regulates accordingly for the choice of materials and restoration procedures.

\section{Conclusion}

This paper proposes a new process of digitization of data concerning chemical products without geometric consistency in a BIM approach. This process manages to go beyond the inaccuracies found in the previous methodologies thanks to the use of an external database capable of sharing data with BIM objects and thus creating a continuously updated synergy. The proposed process is configured as a tool that, in addition to managing data and providing it throughout the process during the phases of interest, acts as a support for the user for the purpose of making choices, guiding him in identifying the products that are suitable for their needs through the simulation of a decision-making process. The process also makes it possible to integrate information relating to the entire life cycle of the products, by setting up a maintenance card integrated with the user's BIM model, which traces its maintenance history by archiving the recovery products used over the years for functionality maintenance. 


\section{References}

Bryde David, Broquetas Martí, Volm Jürgen Marc (2013) The project benefits of Building Information Modelling (BIM). Int J Project Manage 31:971-980

Cavalcante C (2014) Multicriteria decision model to support building maintenance planning. Advanc Mater Res 1468-1477

Charehzehi Aref, Chai ChangSaar, Yusof Aminah Md, Chong Heap-Yih, Loo Siaw Chuing (2017) Building information modeling in construction conflict management. Int J Eng Business Manag 9:1-18

Clough RH, Sears GA, Sears SK (2008) Construction Project Management: A Practical Guide to Field Construction Management

Fu R, Zhang J (2014) Social Involvement to Empower a Better BIM Content Library. Computing in Civil and Building Engineering, pp. 9-16

Kifokeris D, Xenidis EY (2017) Constructability: outline of past, present, and future research. Jd construction engineering and management, vol. 143, n. 8

Miettinen R, Paavola S (2014) Beyond the BIM utopia: approaches to the development and implementation of building information modeling. Autom Constr 43:84-91

National Institute of building sciences (NIBS). United States National Building Information model standard (2018)

OICE, Annual report on the 2018 BIM competition for public works

Sinnamon RM, Andrews JD (1997) Improved accuracy in quantitative fault tree analysis. Qual Reliab Eng Int 13:285-292

Succar B (2009) Building information modelling framework: a research and delivery foundation for industry stakeholders. Automat Construct 18(3):357-375

Taxén L, Lilliesköld J (2008) Images as action instruments in complex projects. Int J Project Manage 26(5):527-536

Tookey J et al (2017) Building Information Modelling (BIM) uptake: clear benefits, understanding its implementation, risks and challenges. Renew Sustain Energy Rev 75:1046-1053

UNI 11337 (2018). Building and civili engineering works- Digital management of the informative. UNI, Ente Nazionale di Normazione

UNI EN 1504 (2006) Products and systems for the protection and repair of concrete structures. Definition, requirements, quality control and evaluation of conformity

UNI EN 934-2 (2012) Admixtures for concrete, mortar and grout. Part 2: concrete admixturesDefinition, requirements, conformity, marking and labelling 
Open Access This chapter is licensed under the terms of the Creative Commons Attribution 4.0 International License (http://creativecommons.org/licenses/by/4.0/), which permits use, sharing, adaptation, distribution and reproduction in any medium or format, as long as you give appropriate credit to the original author(s) and the source, provide a link to the Creative Commons license and indicate if changes were made.

The images or other third party material in this chapter are included in the chapter's Creative Commons license, unless indicated otherwise in a credit line to the material. If material is not included in the chapter's Creative Commons license and your intended use is not permitted by statutory regulation or exceeds the permitted use, you will need to obtain permission directly from the copyright holder.

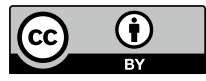

